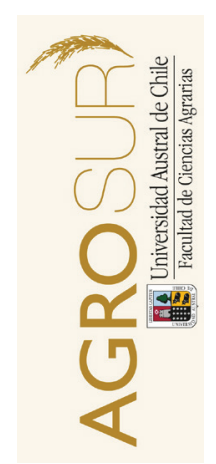

\title{
Control de intensidad de defoliación sobre la producción de forraje estacional y total en sistemas lecheros
}

\author{
Control of defoliation intensity on the seasonal and total forage \\ production in dairy systems
}

\author{
Zibil, S. ${ }^{* a}$, Zanoniani, R. $^{a}$, Bentancur, $0 .^{a}$, Ernst, O. $^{a}$, Chilibroste, P.*a \\ ${ }^{a}$ Estación Experimental “Dr. M. A. Cassinoni”, Facultad de Agronomía, Universidad de la República. \\ Ruta 3, km 363 Paysandú.
}

\begin{tabular}{l} 
A R T I C L E I N F O \\
\hline Article history: \\
Received 02.06.2016 \\
Accepted 19.10.2016 \\
\hline Keywords: \\
Grazing management \\
Post-grazing height \\
Forage yield \\
\hline Original Research Article, \\
Special Issue: Pastures for \\
Sustainable Productions Systems \\
\hline *Corresponding author: \\
Sandra Zibil, Pablo Chilibroste \\
E-mail address: \\
sandra.zibil@gmail.com, \\
pchili@fagro.edu.uy
\end{tabular}

ARTICLE INFO

\author{
A B S T R A C T
}

This study aimed to determine the effect of controlling the intensity of defoliation (ID) on forage production and herbage disappearance in eight commercial farms, in a complete randomized block design. Two blocks were selected in each farm to compare, the grazing management routine of the farm (T0) vs. controlled management (T1, pre grazing height $15-20 \mathrm{~cm}$ and post grazing height $5-7 \mathrm{~cm}$ ). Pre grazing sward height (SH), herbage mass ( $\mathrm{HM}, \mathrm{kg}$ DM ha $\left.{ }^{-1}\right)$ and daily growth rate (GR, $\left.\mathrm{kg} \mathrm{DM} \mathrm{ha}^{-1} \mathrm{~d}^{-1}\right)$ were determined. In average, SH was lower $(p<0.05)$ in autumn (A) and winter (W) $(15.5 \pm 1.2$ and $16.9 \pm 1 \mathrm{~cm}$ ) than in spring (Sp) and summer (Su) $(19.8 \pm 1.0$ and $21.1 \pm 1.2 \mathrm{~cm})$. The same trend ( $\mathrm{p}<0.05)$ followed HM (A: $1250 \pm 164$; W: $1476 \pm 145$; Sp: $1914 \pm 140$; Su: $\left.2564 \pm 167 \mathrm{~kg} \mathrm{DM} \mathrm{ha}^{-1}\right)$. Average GR was $26.5 \%$ higher in T1 than in T0 ( $\mathrm{p}<0.05)$, and significant differences remained throughout the seasons (A: $19.3 \pm 4.3$ vs $7.0 \pm 3.9$; W: $21.3 \pm 3.4$ vs $17.0 \pm 3.0$; Sp: $39.6 \pm 9.0$ vs 33.4 $\pm 2.5 \mathrm{Su}: 43.5 \pm 4.2$ vs $39.7 \pm 4,0 \mathrm{~kg} \mathrm{DM} \mathrm{ha}^{-1} \mathrm{~d}^{-1} ; \mathrm{p}<0.05$, for T1 and T0 respectively). In average, $\mathrm{T} 1$ yielded $2336 \mathrm{~kg} \mathrm{DM} \mathrm{ha}^{-1}$ extra when compared to $\mathrm{T} 0,52 \%$ of that was produced in $\mathrm{A}(\mathrm{p}<0.05)$. Herbage allowance (HA), HM, length of the grazing and $\mathrm{SH}$ explained most of the variation $\left(\mathrm{R}^{2}=0.75\right)$ of the disappeared forage per hectare (offered - rejected). Controlled grazing management increased herbage production and utilization by dairy cows.

\section{RESUMEN}

El estudio tuvo como objetivo determinar el efecto de controlar la intensidad de defoliación (ID) sobre la producción y desaparición de forraje en ocho predios comerciales, con un diseño de bloques completos al azar. Por predio se seleccionaron dos tipos de pasturas y dentro de cada pastura seleccionada se asignaron los tratamientos de ID: T0 (testigo: rutina del predio) y T1 (control de altura de entrada a la pastura 15-20 y salida 5-7 cm). Se determinó la altura del forraje disponible (H, $\mathrm{cm}$ ), disponibilidad de forraje (FD, $\mathrm{kg}$ MS ha-1) y tasa de crecimiento (TC, $\mathrm{kg} \mathrm{MS} \mathrm{ha-1} \mathrm{d}^{-1}$ ). Independiente del tratamiento realizado, la $\mathrm{H}$ fue menor $(\mathrm{p}<0,05)$ en otoño (O) e invierno (I) $(15,5 \pm 1,2$ y 16,9 $\pm 1 \mathrm{~cm})$ que en primavera (P) y verano (V) $(19,8 \pm 1,0 \mathrm{y}$

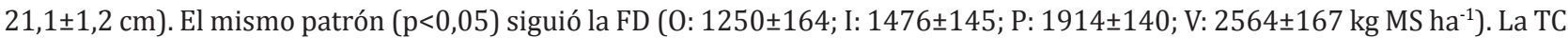
en T1 fue un $26,5 \%$ mayor que T0 (p<0,05), siendo mayor en todas las estaciones del año (0: $19,3 \pm 4,3$ vs 7,0 $\pm 3,9 ; \mathrm{I}: 21,3 \pm 3,4$ vs $17,0 \pm 3,0$; P: $39,6 \pm 9,0$ vs $33,4 \pm 2,5$ y V: $43,5 \pm 4,2$ vs $39,7 \pm 4,0 ; p<0,05$, respectivamente). El efecto de T1 sobre la producción de forraje anual fue de $2336 \mathrm{~kg}_{\mathrm{MS}} \mathrm{ha}^{-1}$ extra y el 0 explicó el 52\% de la diferencia total de forraje anual. La asignación de forraje (AF, cantidad de forraje promedio ofrecido por vaca y por día), horas de acceso al pastoreo, FD y H del forraje explicaron una alta proporción $\left(\mathrm{R}^{2}=0,75\right)$ del forraje desaparecido por ha $(\mathrm{D}=$ ofrecid - residual). El control del pastoreo incrementó la producción y la eficiencia de utilización de forraje.

Palabras clave: manejo del pastoreo, altura remanente, producción de forraje.

\section{INTRODUCCIÓN}

La pastura constituye un componente clave de los sistemas de producción de leche si se tiene en cuenta que es el alimento de menor costo por unidad de materia seca (MS) o energía metabolizable (EM) (Dillon et al., 2005; Chilibroste et al., 2010). Sin embargo, los resultados bio-económicos dependen de la estrategia de alimentación implementada y sus efectos sobre el consumo total de MS, el consumo de forraje y su eficiencia de utilización. Trabajos previos han analizado la producción y utilización de pasturas con el objetivo de incrementar productividad, aunque la misma está fuertemente condicionada por la habilidad del sistema implementado de producir y cosechar la biomasa requerida para cubrir los requerimientos de los animales a lo largo 
del año (Mayne et al., 2000; Dillon et al., 2005; Wales et al., 2005; Rossi y García, 2006). El aumento de la carga con el objetivo de aumentar la productividad animal por unidad de superficie de los sistemas lecheros puede provocar intensas defoliaciones con un impacto negativo en la cantidad total de forraje producido (García y Fariña, 2010). Adicionalmente, el incremento en la frecuencia de pastoreo, principalmente en épocas donde las TC son lentas, compromete la persistencia en el tiempo de las pasturas, deprimiendo la tasa de rebrote y sobrevivencia de plantas (Zanoniani, 2010; Formoso, 2009).

Antecedentes en Uruguay (Chilibroste et al., 2003) indican que en el área de vaca masa (VM) de 37 predios remitentes a Conaprole (Cooperativa Nacional de Productores de Leche) la producción de forraje cosechado no superó los $3200 \mathrm{Kg} \mathrm{MS} \mathrm{ha-1}$. La condición de las pastura (FD y H) en el área destinada a las VO (vacas en ordeñe) durante el período 0-I fue limitante para una eficiente cosecha por parte de los animales indicando que los valores de utilización del forraje (45$50 \%$ ) estuvieron determinados por la estructura de la pastura (Chilibroste et al., 2003). Adicionalmente, los valores de FD y $\mathrm{H}$ remanente condicionaron la tasa de rebrote de la pastura deprimiendo la producción total de forraje. Esto puso en evidencia una serie de factores de la pastura, como limitantes para mejorar los niveles de cosecha de forraje en sistemas de producción de leche donde la pastura es la fuente principal de alimentación. Más recientemente Chilibroste y Battegazzore (2014), en un programa de monitoreo de sistemas comerciales de producción de leche en el que se determinó mensualmente el margen de alimentación (MA) como indicador del estado de resultado del sistema de producción, indicaron que en la mayoría de los predios donde el MA se ubicó entre 3-3,5 U\$S ha vaca ${ }^{-1}$ día $^{-1}$, la producción de forraje en el área de VM fue del orden de $6000 \mathrm{~kg} \mathrm{MS} \mathrm{ha}^{-1}$, y las estimaciones de consumo de forraje se ubicaron en $3200 \mathrm{Kg}$ MS con una utilización estimada del 50\%. En estas condiciones, la información es consistente en que las medidas de manejo que se apliquen en el control de la ID constituyen herramientas relevantes para incrementar la producción y persistencia de forraje, y maximizar la conversión a producto animal (Pérez-Prieto y Delagarde, 2012; Parga et al., 2000; Rook, 2000). Al definir estrategias de manejo a nivel de pastura, se debe considerar además de la producción de forraje otras características que afectan la accesibilidad del forraje por parte del animal como biomasa aérea, altura de follaje (variable que determina el tamaño de bocado del animal), y tamaño de lámina (Prache y Peyraud, 1997). Aun así, existen limitantes para mejorar la producción de forraje y su utilización si no se comprende la forma en que se cosecha el forraje y el manejo del área foliar, dado que determinan la TC y la producción total de MS (Parsons y Chapman, 2000; Parsons, 1988). En sistemas de pastoreo rotati- vo el control de AF es una medida de manejo que incide directamente en el consumo de los animales. La relación entre la AF y consumo de materia seca (CMS) ha sido bien establecida para un rango amplio de $\mathrm{AF}$ (Delagarde y O'Donovan, 2005). Sin embargo el manejo de la AF no es una variable fácil de implementar a nivel de predios lecheros, y puede ser facilitada, si se incluye el manejo de la altura de remanente post pastoreo (expresada en proporción a la altura previo al pastoreo) lo que define la ID, siendo este un buen indicador de AF y CMS (Delagarde et al., 2001).

El objetivo de este estudio fue determinar el efecto del control de la ID sobre la producción y cosecha de forraje por parte de VO en sistemas comerciales de producción de leche. La hipótesis establecida fue que el control de la ID (altura de entrada y remanente durante la sesión de pastoreo) promueve favorablemente la TC de la pastura y la producción estacional y total de forraje.

\section{MATERIALES Y MÉTODOS}

\section{Localización y periodo experimental}

El estudio se llevó a cabo durante el período febrero-2003 a febrero-2005 en ocho predios comerciales de la cuenca lechera del Sur de Uruguay remitentes a CONAPROLE (Florida, San José, Canelones, Soriano y Maldonado). Las zonas seleccionadas se caracterizan por la dominancia de suelos de tipo Vertisoles Rúpticos Lúvicos (típicos) y Brunosoles eutricos y subeutricos típicos/lúvicos (MGAP-Renare, 1976).

\section{Selección de Potreros}

El experimento se desarrolló sobre la base y rotación forrajera que cada productor tenía implementada en su predio. Se consideró para la selección de los sitios experimentales el estado de la pastura, la edad y tipo de pastura utilizada al inicio del experimento (febrero 2003) y la cercanía a la sala de ordeñe y/o la frecuencia de uso para pastoreo en el área de VO. En cada predio se seleccionaron dos potreros, uno de los cuales tenía una pastura en plena producción de segundo y/o tercer año (P2 oP3) y el otro una pastura recién implantada (P1) o cultivo forrajero de invierno (Vi) (cuadros 1 y 2); las mismas se monitorearon simultáneamente en el tiempo. Se buscó que los potreros seleccionados por predio fueran lo más homogéneos posibles en cuanto a tipo de suelo y topografía.

En cada uno de los potreros se registraron las prácticas agronómicas tales como fecha de siembra, fertilización de pasturas, manejo del pastoreo y mezcla forrajera. Para cuantificar la producción de forraje se determinó TC mediante jaulas móviles de exclusión (Berreta et al., 1993). 
Cuadro 1. Principales mezclas y densidad a la siembra de pasturas de segundo y/o tercer año utilizadas y monitoreadas por predio.

Table 1. Major mixtures and sowing density of pastures used and monitored in each farm, at second and/or third year.

\begin{tabular}{clcc}
\hline Predio & \multicolumn{1}{c}{$\begin{array}{c}\text { Mezcla } \\
\text { forrajera }\end{array}$} & $\begin{array}{c}\text { Dosis } \\
\left(\mathrm{kg} \mathrm{ha}^{-1}\right)\end{array}$ & $\begin{array}{c}\text { Fecha de } \\
\text { siembra }\end{array}$ \\
\hline 1 & TR.TB.AA.Rg. & $6+5+10+10$ & $20-03-2002$ \\
2 & TR.TB.Lo.Rg. & $8+1+6+15$ & $20-04-2002$ \\
3 & TR.TB.Rg.Festuca & $14+1+10+10$ & $27-06-2002$ \\
4 & TR.TB.Lo.Rg. & $4+2+10+15$ & $04-03-2003$ \\
5 & TR.Rg. & $8+15$ & $14-04-2003$ \\
6 & AA.Lo.Rg. & $10+15+15$ & $28-04-2002$ \\
7 & TR.Lo. & $8+10$ & $20-04-2002$ \\
8 & AA.Dact.Av. & $13+12+50$ & $3-04-2002$ \\
\hline
\end{tabular}

TR: Trébol rojo, Trifolium pratense; TB: Trébol blanco, Trifolium repens; Lo: Lotus corniculatus; AA: Medicago sativa; Rg: Raigrás, Lolium multiflorum; Fest: Festuca arundinacea; Dact: Dactylis glomerata; Av: Avena sativa; Ach: Chicoria: Cichorium Intybus.

Cuadro 2. Principales mezclas y densidad a la siembra de pasturas de primer año y cultivo forrajeros utilizadas y monitoreados por predio.

Table 2. Main mixtures and sowing density of first year pastures and annual forage used and monitored in each farm.

\begin{tabular}{|c|c|c|c|}
\hline Predio & $\begin{array}{c}\text { Mezcla } \\
\text { forrajera }\end{array}$ & $\begin{array}{c}\text { Dosis } \\
\left(\mathrm{kg} \mathrm{ha}^{-1}\right)\end{array}$ & $\begin{array}{l}\text { Fecha de } \\
\text { siembra }\end{array}$ \\
\hline 1 & TR.TB.AA.Rg. & $6+5+10+15$ & 20-04-2003 \\
\hline 2 & $\begin{array}{l}\text { TR.TB.Lo.Ach. } \\
\text { Rg.anual Rg. } \\
\text { Bianual }\end{array}$ & $7+1+7+6+5+5$ & 28-03-2003 \\
\hline 3 & TR.TB.Rg.Fest. & $8+1+8+10$ & 04-04-2003 \\
\hline 4 & Av. & 100 & $21-02-2003$ \\
\hline 5 & TR.Lo.Fest.Av. & $10+10+12+40$ & $12-04-2004$ \\
\hline 6 & AA.Lo.Rg. & $10+15+15$ & $29-05-2003$ \\
\hline 7 & TB.AA.Dact.Rg. & - & 20-04-2002 \\
\hline 8 & Av. & 100 & $22-02-2002$ \\
\hline
\end{tabular}

TR: Trébol rojo, Trifolium pratense; TB: Trébol blanco, Trifolium repens; Lo: Lotus corniculatus; AA: Medicago Sativa; Rg: Raigrás, Lolium multiflorum; Fest: Festuca arundinacea; Dact: Dactylis glomerata; Av: Avena sativa; Ach: Chicoria: Cichorium Intybus.

\section{Tratamientos y diseño experimental}

El diseño del experimento fue de bloques completos al azar. En cada predio se seleccionaron dos bloques (dentro de las pasturas seleccionadas) y dentro de cada uno de los bloques se sortearon los dos tratamientos. En cada potrero se marcó un área lo más homogénea posible y representativa del mismo, correspondiente a un $30 \%$ del total (bloque), que a su vez fue subdividida en dos parcelas y en las que se asignaron los tratamientos:

Tratamiento testigo (T0): el productor aplica los criterios habituales de manejo del pastoreo.

Tratamiento manejo controlado (T1): Se aplican las prácticas agronómicas definidas para controlar la ID en base a dos criterios: altura del forraje al ingreso del pastoreo $(15-20 \mathrm{~cm})$ con cobertura total del suelo y altura de forraje de salida o remanente $(5-7 \mathrm{~cm})$.

\section{Mediciones en la pastura}

\section{Tasa de crecimiento}

La determinación de la TC se estimó mediante la utilización de jaulas de exclusión móviles, de acuerdo a la descripción de Berretta et al. (1993). Al inicio del experimento y previo a la colocación de las jaulas se determinó en cada parcela el FD inicial. Para los casos en que se realizaron determinaciones en $\mathrm{P} 1$ o Vi, el inicio del experimento estuvo determinado por el FD alcanzado para iniciar el primer pastoreo, mientras que en el caso de las pasturas de más edad (P2, P3) el inicio del experimento se determinó en función de la secuencia de pastoreo ya instalada. Se instalaron tres jaulas $(1 \mathrm{~m}$ $\mathrm{x} 1 \mathrm{~m}$ ) por parcela.

La frecuencia entre cortes dependió de la entrada y salida de los pastoreos en cada parcela y estación del año, la cual fue controlada en función del FD del área de pastoreo.

\section{Altura y disponibilidad}

Para los cortes dentro de la jaula se utilizó un marco rectangular de $0,20 \times 0,50 \mathrm{~cm}$. Previamente a los cortes dentro de la jaula, se determinó la altura de forraje en cinco puntos por cuadro (usando una regla graduada registrando el punto de la hoja viva más alta sin extender que tocara la regla), con el objetivo de determinar las relaciones entre altura y biomasa aérea de forraje tanto para el forraje disponible como para el forraje remanente (Haydock y Shaw, 1975). Los cortes se realizaron a ras del piso con tijera de aro manual de esquilar. Las estaciones se definieron según Berretta et al. (1993): V (diciembre, enero y febrero), 0 (marzo, 
abril y mayo), I (junio, julio y agosto) y P (setiembre, octubre y noviembre). Previo al ingreso de los animales a la pastura asignada se determinó la AF (Kg MS vaca $^{-1} \mathrm{~d}^{-1}$ ) en función de la disponibilidad de forraje promedio ofrecido por vaca y por día ajustando el área de franja diaria ofrecida. El tiempo de pastoreo fue determinado desde el momento que ingresaban a la pastura asignada y hasta alcanzar la altura de forraje remanente entre 7-5 cm. El forraje desaparecido (D) se determinó por diferencia entre Kg de MS ofrecido y Kg de MS remanente.

\section{Modelos y análisis estadístico}

La información de las TC, FD y H se analizó con un modelo mixto de medidas repetidas en el tiempo, con el uso del procedimiento GLIMMIX (SAS, 2010). El modelo incluyó los efectos fijos del tipo de forraje, tratamiento, estación y las interacciones forraje*tratamiento y tratamiento*estación (Ecuación 1). El modelo incluyó el efecto bloque como efecto aleatorio. La unidad experimental sobre la que se realizaron las medidas repetidas en el tiempo fue la parcela. Se analizó la estructura de covarianza utilizando una estructura de primer orden heterogénea y autorregresiva ARH (1) en base al criterio de Akaike. Las medidas se compararon usando la prueba de Tukey y los efectos se reportaron como significativos cuando $\mathrm{P}<0,05$ y como tendencia con valores de $\mathrm{P}>0,05 \mathrm{y}<0,1$.

$$
\begin{aligned}
& \text { Yijk }=\mu+T_{i}+\beta_{j}+\text { efecto forraje }+ \text { efecto estación }+ \\
& \text { (forraje*tratamiento }_{k i}+\left(\text { tratamiento }^{*} \text { estación }\right)_{i l}+\boldsymbol{\varepsilon}_{i j k l}
\end{aligned}
$$

Para estudiar la relación entre FD y $\mathrm{H}$ de la pastura (P1, P2 y P3) en los diferentes años, se utilizó un modelo de regresión lineal simple utilizando el procedimiento PROC REG SAS (SAS, 2010) (Ecuación 2).

$$
Y i=\beta_{0}+\beta_{1 x i}+\varepsilon_{i}
$$

Dónde: $Y i$ es la variable de respuesta FD por ha, $\beta_{0}$ el intercepto del modelo, $\beta_{1}$ el coeficiente de regresión lineal de la variable ${ }_{X i}$ altura de forraje en $\mathrm{cm}$. El coeficiente de determinación $\mathrm{R}^{2}$ se utilizó para determinar la bondad de ajuste del modelo. El valor crítico $\mathrm{F}$ se reportó como significativos cuando $\mathrm{P}<0,05$ y como tendencia con valores de $\mathrm{P}>0,05 \mathrm{y}<0,1$.

Para cuantificar la relación entre D y las variables AF, tiempo de acceso a la pastura (horas), FD y $\mathrm{H}$, se utilizó un modelo de regresión múltiple utilizando el procedimiento PROC REG con el método de selección stepwise (SAS, 2010).

\section{RESULTADOS Y DISCUSIÓN}

\section{Disponibilidad y altura de la pastura}

Los valores medios de FD total para los diferentes tipos de forraje analizado (P1, P2, P3) no fueron significativamente diferentes $(\mathrm{P}>0,05)$, mientras que $\mathrm{H}$ mostró una tendencia $(\mathrm{P}<0,1)$ a ser mayor en $\mathrm{P} 1 \mathrm{y}$ Vi con respecto a P2 y P3 (Cuadro 3).

Los valores de disponibilidad determinados al inicio de cada pastoreo, registraron un valor máximo de 1919 kg MS ha-1 para Vi. Si bien no se detectaron diferencias significativas, el valor mayor en las pasturas fue observado en P1, registrando un 7\% más de disponibilidad con respecto a P2 y 11\% más que P3 (Cuadro 3).

Los cultivos forrajeros de invierno que lograron iniciar los pastoreos en 0 alcanzaron valores de FD más altos que el resto de las pasturas de diferentes edades indicando que son una buena opción para tener adecuada FD en el invierno y primavera (Formoso, 2009). El crecimiento rápido e inicial de los Vi y las P1 que van asociadas a verdeos permitirían lograr niveles de FD mayores a $1500 \mathrm{~kg} \mathrm{MS} \mathrm{ha-1}^{-1}$ que definirían la decisión de ingresar al pastoreo. Esta ventaja en el corto plazo ha sido una de las estrategias seguidas por los productores y corroborada en DIEA (2014) apreciando una evolución creciente del porcentaje de superficie sembrada a favor de forrajeras anuales (Vi) en un fuerte proceso de anualización de la estructura forrajera.

En el Cuadro 4 se presenta los valores de FD según la estación de crecimiento. El crecimiento de la pastura en el período 0 -I registró valores promedio de FD

Cuadro 3. Efecto del tipo de forraje (componente) sobre el forraje disponible y la altura del forraje a la entrada del pastoreo en ocho predios lecheros monitoreados en la cuenca sur de Uruguay (febrero 2003 - febrero 2005) (promedio \pm desviación estándar).

Table 3. Effect of forage type (component) on average availability (FD) and height $(\mathrm{H}$ in $\mathrm{cm})$ at the entrance of grazing in eight dairy farms monitored in the south of Uruguay (February 2003 - February 2005) (mean \pm standard deviation).

\begin{tabular}{ccc}
\hline Componente & FD Kg Ms ha $^{-1}$ & H cm \\
\hline P1 & $1.869 \pm 131^{\mathrm{a}}$ & $18,07 \pm 0,9^{\mathrm{a}}$ \\
P2 & $1.748 \pm 106^{\mathrm{a}}$ & $16,5 \pm 0,7^{\mathrm{b}}$ \\
P3 & $1.669 \pm 178^{\mathrm{a}}$ & $16,0 \pm 1,3^{\mathrm{b}}$ \\
Vi & $1.919 \pm 278^{\mathrm{a}}$ & $22,6 \pm 2,25^{\mathrm{a}}$ \\
\hline
\end{tabular}

P1=pastura de primer año, P2=pastura de segundo año, P3=pastura de tercer año, $\mathrm{Vi}=$ cultivo forrajero de invierno. Literales diferentes denotan una tendencia de $\mathrm{P}<0,1$.

FD: forraje disponible. $\mathrm{H}$ : altura en $\mathrm{cm}$. 
por debajo de los $1.500 \mathrm{~kg} \mathrm{MS} \mathrm{ha}^{-1}$ fijados como criterio para el inicio del pastoreo. A pesar de que la $\mathrm{H}$ no fue limitante para la entrada al pastoreo, en 0 registró los menores valores.

En P y V $\left(1914 \pm 140\right.$ y $2564 \pm 167, \mathrm{~kg} \mathrm{MS} \mathrm{h}^{-1}$ respectivamente) se alcanzaron los valores más altos de FD al ingreso del pastoreo indicando un período más favorable en la producción de forraje que permitió superar en un $64 \%$ la oferta de forraje en 0 .

La marcada estacionalidad de las pasturas sembradas en Uruguay hace que la eficiencia de utilización de éstas tome una particular importancia, principalmente en el O-I. En esta época la principal limitante en la respuesta productiva animal es el desbalance entre la oferta y demanda total de nutrientes (Chilibroste, 2011), por lo tanto las condiciones de disponibilidad que las pasturas en el período 0-I sugieren que el sistema no puede ser sostenido sólo a pasto y es necesario estrategias de alimentación que integren la suplementación con forraje conservado y/o concentrados para cubrir estos desbalances y a su vez evitar situaciones de sobrepastoreo (Formoso, 2008). Antecedentes en la misma línea de trabajo reportados por Chilibroste et al. (2003) expresaron que los valores de FD al igual que los de $\mathrm{H}$, declinaron linealmente entre los meses de abril a julio recuperándose luego en primavera.

En promedio, la $\mathrm{H}$ al ingreso del pastoreo no mostró diferencias significativas $(P>0,05)$ entre tratamientos, (18,6 vs $18,0 \mathrm{~cm}$, para T1 y T0 respectivamente). Independientemente del tratamiento, la altura al ingreso de las P2 y P3 no difieren entre sí (16,5 vs 16,0 respectivamente, $P>0,05)$, pero fueron significativamente más bajas que $\mathrm{P} 1$ y Vi $(18,07$ y $22,6 \mathrm{~cm}$ respectivamente, $\mathrm{P}<0,05)$.

\section{Efecto de la interacción tipo de forraje por trata-} miento

Cuando analizamos la interacción tipo de pastura por tratamiento, se observó en las P2 una diferencia significativa $(\mathrm{P}<0,05)$ en $\mathrm{H}$ para $\mathrm{T} 1$ respecto de T0 $(17,2$ vs $15,8 \mathrm{~cm}$, respectivamente). La altura de ingreso para las P2 fue significativamente menor lo que estaría indi- cando una mayor frecuencia de uso y un menor tiempo de recuperación, sugiriendo una tendencia al sobrepastoreo, probablemente vinculado con la menor área disponible para pastoreo de P1 y Vi.

\section{Tasas de crecimiento y producción de forraje}

En el Cuadro 5 se presentan las TC de las pasturas para ambos tratamientos y tipos de forraje (P1, P2, P3 y Vi). Las TC promedio en T1 fueron significativamente superiores (26\% más) respecto a T0, esa diferencia entre tratamientos se mantuvieron para todos sus componentes (P1, P2, P3 y Vi).

El tipo de forraje dentro de cada tratamiento muestra para T1 que P1 y Vi tuvieron TC más altas que P2 y

Cuadro 5. Tasa de crecimiento ( $k g$ MS ha- $\mathrm{d}^{-1}$ ) total y por tipo de forraje (promedios \pm desviacíon estándar) por tratamiento durante el período experimental. T0: tratamiento testigo (rutina de pastoreo del predio) y T1: tratamiento controlado (control de altura de entrada a la pastura 15-20 y salida 5-7 cm).

Table 5. Growth rates (kg DM ha $\left.{ }^{-1} \mathrm{~d}^{-1}\right)$ and by type of forage (mean \pm standard deviation) per treatment. T0: control (farm's grazing management routine); T1: controlled management (pre grazing height $15-20 \mathrm{~cm}$ and post grazing height 5-7 cm).

\begin{tabular}{ccc}
\hline & T0 & T1 \\
\hline Promedio Total & $24,5 \pm 2,3^{\mathrm{b}}$ & $30,9 \pm 2,5^{\mathrm{a}}$ \\
\hline Componente & \\
\hline P1 & $27,0 \pm 2,9^{\mathrm{b}, \mathrm{y}}$ & $35,7 \pm 3,3^{\mathrm{a}, \mathrm{y}}$ \\
P2 & $20,5 \pm 2,0^{\mathrm{b}, \mathrm{y}}$ & $25,8 \pm 2,2^{\mathrm{a}, \mathrm{z}}$ \\
P3 & $21,0 \pm 3,8^{\mathrm{b}, \mathrm{y}}$ & $25,7 \pm 4,1^{\mathrm{a}, \mathrm{z}}$ \\
Vi & $29,5 \pm 6,6^{\mathrm{b}, \mathrm{x}}$ & $36,7 \pm 7,3^{\mathrm{a}, \mathrm{y}}$ \\
\hline
\end{tabular}

a,b: denotan diferencias significativas entre columnas; $\mathrm{x}, \mathrm{y}, \mathrm{z}$ : denotan diferencias estadísticas entre filas $(\mathrm{P}<0,05)$.

Cuadro 4. Efecto de la estación de crecimiento sobre el forraje disponible y la altura en cm del total de forraje durante todo el período experimental (promedio \pm desviación estándar).

Table 4. Effect of the growing season on FA $\left(\mathrm{kg} \mathrm{DM} \mathrm{ha}^{-1}\right)$ and $\mathrm{SH}$ in $\mathrm{cm}$ of total forage throughout the experimental period (mean \pm standard deviation).

\begin{tabular}{ccccc}
\hline & 0 & $\mathrm{I}$ & $\mathrm{P}$ & $\mathrm{V}$ \\
\hline${\mathrm{FD}\left(\mathrm{Kg} \mathrm{MS} \mathrm{ha}^{-1}\right)}$ & $1250 \pm 164^{\mathrm{d}}$ & $1476 \pm 145^{\mathrm{c}}$ & $1914 \pm 140^{\mathrm{b}}$ & $2564 \pm 167^{\mathrm{a}}$ \\
$\mathrm{H}(\mathrm{cm})$ & $15.5 \pm 1.2^{\mathrm{d}}$ & $16,9 \pm 1,06^{\mathrm{c}}$ & $19,8 \pm 1,03^{\mathrm{b}}$ & $21,08 \pm 1,2^{\mathrm{a}}$ \\
\hline
\end{tabular}

O: otoño, I: invierno, P: primavera, V: verano. Literales diferentes denotan diferencias significativas ( $\mathrm{p}<0,05)$. FD: forraje disponible. H: altura en cm. 


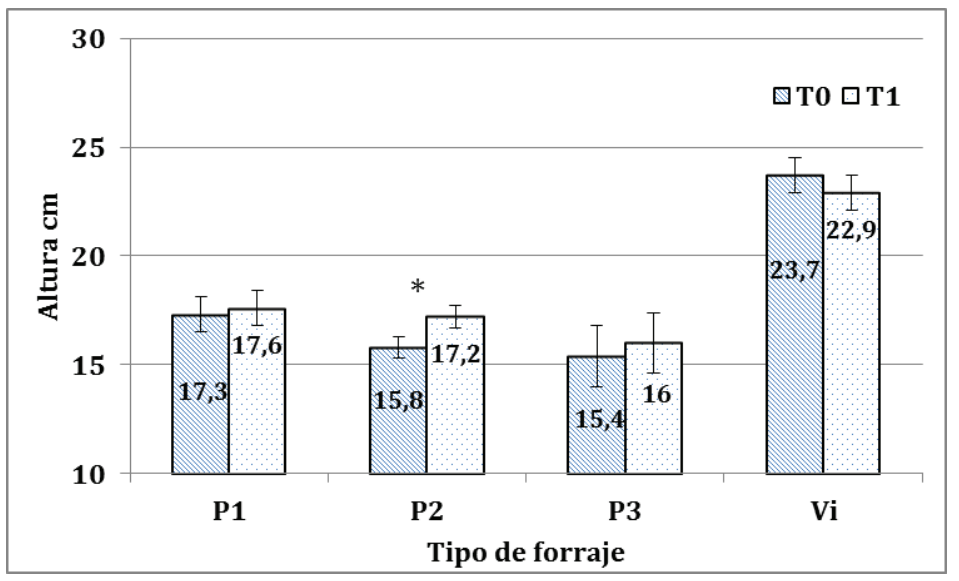

Figura 1. Resultados de altura de forraje según tipo de forraje y tratamiento.

Figure 1. Forage height results according to type of forage and treatment.

*: Denota diferencias significativas $(\mathrm{P}<0,05)$ entre tratamientos. T0: tratamiento testigo (rutina de pastoreo del predio); 1 : tratamiento controlado (control de altura de entrada a la pastura 15-20 y salida 5-7 cm).

*: Denotes statistical significant differences. T0: control (grazing management routine of the farm); T1: controlled management (pre grazing height $15-20 \mathrm{~cm}$ and post grazing height 5-7 cm).

P3 $(\mathrm{P}<0,05)$; para T0 sólo fue significativamente mayor $(\mathrm{P}<0,05)$ para Vi respecto del resto. A su vez, en T1 no hubieron diferencias significativas $(\mathrm{P}>0,05)$ entre $\mathrm{P} 1 \mathrm{y}$ Vi y tampoco entre P2 y P3 indicando patrones de crecimiento de forraje similar (Cuadro 5).

En todas las estaciones del año, el T1 superó al T0 y en otoño se registraron las diferencias más altas, alcanzando el T1 valores de TC de $170 \%$ por encima del $\mathrm{T} 0$, lo que indica el impacto en el manejo del pastoreo en las épocas más críticas de crecimiento del forraje (Cuadro 6).

Las diferencias promedio de TC entre T0 y T1 alcanzan a 6,4 $\mathrm{kg} \mathrm{MS} \mathrm{d}^{-1}$, que en el año implican una producción extra de $2336 \mathrm{~kg} \mathrm{MS} \mathrm{ha}^{-1} \mathrm{año}^{-1}$ (Cuadro 5). El análisis de las TC por tratamiento y por estación indican que la producción extra de T1 esta explicada en un $52 \%$ por la diferencia generada en el 0 (1107 Kg MS ha $^{-1}$ ) (Cuadro 6).

El 0 registró en ambos tratamientos los menores valores de TC indicando un potencial de crecimiento de las pasturas significativamente menor con respecto al periodo primavera-verano, demostrando valores que no permiten alcanzar el objetivo de los $1500 \mathrm{~kg}$ MS $\mathrm{ha}^{-1}$ de FD recomendados para iniciar el pastoreo. En el invierno las TC alcanzaron valores un $48 \%$ superior al 0 , indicando mejores condiciones de crecimiento y establecimiento de la pastura.

Analizando la distribución de producción de forraje anual por período, el O-I produjo el $29 \%$ del forraje total con respecto al 70,1\% producido en el período P-V.

La dinámica de cosecha y el manejo del forraje remanente para las diferentes especies de la pastura, en las diferentes estaciones del año y particularmente en el 0 , afectó positivamente la TC y por lo tanto la producción de MS total posible de ser cosechada por los animales. Tal como indicaron Parsons y Chapman (2000), el estado residual de la pastura o condición inicial de rebrote en éste estudio afectó la TC, lo que podría explicar que, pese a las TC en 0 fueron inferiores con respecto a las de $P(13,2 \pm 3,7$ vs. $36,6 \pm 2,5$, respectivamente), el T1 permitió alcanzar una mejor condición de la

Cuadro 6. Tasa de crecimiento (kg MS ha-1 $\left.\mathrm{d}^{-1}\right)$ según tratamiento, T0: tratamiento testigo (rutina de pastoreo del predio) y T1: tratamiento controlado (control de altura de entrada a la pastura 15-20 y salida 5-7 cm), y estación del año (promedio \pm desvío estándar).

Table 6. Growth rates (kg DM ha- $\mathrm{d}^{-1}$ ) per treatment (T0: control, grazing management routine of the farm; T1: controlled management, pre grazing height $15-20 \mathrm{~cm}$ and post grazing height $5-7 \mathrm{~cm}$ ), and season (mean \pm standard deviation).

\begin{tabular}{ccc}
\hline Estación & T0 & T1 \\
\hline O & $7,0 \pm 3,9^{\mathrm{b}, \mathrm{z}}$ & $19,3 \pm 4,3^{\mathrm{a}, \mathrm{y}}$ \\
I & $17,8 \pm 3,0^{\mathrm{b}, \mathrm{y}}$ & $21,3 \pm 3,4^{\mathrm{a}, \mathrm{y}}$ \\
P & $33,4 \pm 2,5^{\mathrm{b}, \mathrm{x}}$ & $39,6 \pm 2,9^{\mathrm{a}, \mathrm{x}}$ \\
V & $39,7 \pm 4,0^{\mathrm{b}, \mathrm{x}}$ & $43,5 \pm 4,2^{\mathrm{a}, \mathrm{x}}$ \\
\hline
\end{tabular}

a,b: denotan diferencias estadísticas entre columnas; $\mathrm{x}, \mathrm{y}$, z: denotan diferencias estadísticas entre filas $(\mathrm{P}<0,05)$. 
pastura con un índice de área foliar (IAF) mayor, mayor tasa de fotosíntesis neta (Parsons y Chapman, 2000) y por lo tanto mayor TC que en T0 $(19,3 \pm 4,3$ vs. $7,0 \pm 3,9)$.

En los sistemas de producción de leche de Uruguay, las mermas productivas de forraje en otoño frecuentemente comienzan a gestarse con el manejo estival de la pastura (excesiva intensidad y frecuencia de defoliación), particularmente en veranos secos. Esto ha sido demostrado por Formoso (2009) quien indicó que, pastoreos frecuentes a la salida del verano y principios de otoño deprimieron la producción de forraje en más de un $40 \%$. El sobrepastoreo en ésta época afectó la persistencia de la pastura, debido al agotamiento de las reservas y en consecuencia, una respuesta menor de rebrote luego del pastoreo. Esto fue demostrado en ese estudio, considerando solamente una secuencia de pastura más un $\mathrm{Vi}$, donde el acortamiento de la rotación forrajera disminuyó la superficie efectiva de pastoreo disponible en V-O provocando el mencionado sobrepastoreo de una pastura de segundo año de Festuca arundinacea Schreb., Trifolium repens L., y Lotus corniculatus $\mathrm{L}$.

\section{Estimación de FD y su relación con la H}

Un componente básico de la estimación de AF es lograr una buena estimación de FD (Chilibroste et al., 2003). Para ello, se buscó establecer la relación entre FD y H como atributo de fácil determinación. El Cuadro 7 presenta las regresiones lineales para P1, P2 y P3.

Las funciones de regresión tienen un coeficiente de determinación alto destacándose $\mathrm{P} 3\left(\mathrm{R}^{2}=0,72\right)$, a pesar del número de observaciones realizadas y la variabilidad de las pasturas analizadas. La similitud de los coeficientes lineales (90-100 Kg MS por cm de altura) para las pasturas coinciden con los reportados por Chilibroste et al. (2003)

\section{Forraje desaparecido}

Se estudió el efecto conjunto de AF, tiempo de acceso al pastoreo, FD y $\mathrm{H}$ sobre el forraje desaparecido por hectárea (D = Kg MS ofrecido - Kg MS residual). Estos factores en conjunto explicaron un $75 \%$ de la variación observada en D de acuerdo a la siguiente ecuación (Ecuación 3):

$$
\begin{aligned}
& \mathrm{D}=-3.79+0,51^{*} \mathrm{AF}\left(\mathrm{kg} \text { MS VO-1 } \mathrm{d}^{-1}\right)+0,14^{*} \\
& \text { tiempo de acceso a la pastura (horas) }+0,10^{*} \mathrm{H}(3) \\
& (\mathrm{cm})+0,002^{*} \mathrm{FD}\left(\mathrm{kg} \mathrm{MS} \mathrm{ha}^{-1}\right),\left(\mathrm{n}=210 ; \mathrm{R}^{2}=0,75\right)
\end{aligned}
$$

El Cuadro 8 resume el orden de las variables que ingresaron a la ecuación y el coeficiente de determinación ( $\mathrm{R}^{2}$ parcial y $\mathrm{R}^{2}$ del modelo) para cada una de las variables que participaron en la misma.

El coeficiente de determinación acumulado resultó alto $\left(\mathrm{R}^{2}=0,75\right)$ y a su vez la $\mathrm{AF}$ explicó el $70 \%$ del $\mathrm{D}$ por lo cual la $\mathrm{AF}$ es un indicador que estima con buena precisión el D. La incorporación del FD, la $\mathrm{H}$ y el tiempo de acceso de las VO a la pastura en la ecuación mejoraron la predicción significativamente aunque con un peso relativo menor. Estos resultados concuerdan con los encontrados por Peyraud et al. (1996) demostrando que si bien el consumo individual de las vacas está mayormente afectado por la asignación de la pastura,

Cuadro 8. Forraje desaparecido: Resumen de las variables seleccionadas para el modelo de regresión múltiple por método de selección stepwise (SAS, 2010).

Table 8. Disappeared forage: Summary of selected variables for the multiple regression model by stepwise selection method (SAS, 2010).

\begin{tabular}{lccc}
\hline \multicolumn{1}{c}{ Variable } & $\mathrm{R}^{2}$ parcial & $\mathrm{R}^{2}$ & $\mathrm{P}$ \\
\hline AF $\left(\right.$ Kg MS vaca $\left.^{-1} \mathrm{~d}^{-1}\right)$ & 0,70 & 0,70 & $\mathrm{P}<0,0001$ \\
FD $\left(\right.$ Kg MS ha $\left.^{-1}\right)$ & 0,0379 & 0,7391 & $\mathrm{P}<0,0001$ \\
Horas pastoreo & 0,0042 & 0,7433 & 0,0681 \\
H & 0,0036 & 0,7469 & 0,0886 \\
\hline
\end{tabular}

AF: asignación de forraje. FD: forraje disponible. H: altura en $\mathrm{cm}$.

Cuadro 7. Relación entre forraje disponible (Kg MS ha-1) y altura (cm) del forraje ofrecido.

Table 7. Relationship between availability $\left(\mathrm{Kg} \mathrm{DM} \mathrm{ha}^{-1}\right)$ and height $(\mathrm{cm})$ of forage offered.

\begin{tabular}{cccc}
\hline Tipo de forraje & Ecuación de regresión & $\mathrm{R}^{2}$ & $\mathrm{P}$ \\
\hline P1 $(\mathrm{n}=65)$ & Kg MS disp. ha ${ }^{-1}=353+90,47^{*}$ altura & 0,40 & $<0,0001$ \\
P2 $(\mathrm{n}=138)$ & Kg MS disp. ha ${ }^{-1}=72,4+100,49 *$ altura & 0,55 & $<0,0001$ \\
P3 (n=26) & Kg MS disp. ha ${ }^{-1}=34,4+95,2 *$ altura & $0,72<0,0001$ \\
\hline
\end{tabular}

P1, P2 y P3: pasturas de $1^{\circ}, 2^{\circ}$ y $3^{\circ}$ año. $R^{2}$ : coeficiente de determinación. 
la estructura original de la pastura (masa y altura de forraje previo al pastoreo) afecta la tasa de consumo, la eficiencia de utilización y el consumo total de MS. En una revisión reciente (Chilibroste et al., 2015) han demostrado el impacto del tiempo de acceso la pastura sobre el tiempo efectivo de pastoreo de los animales y en consecuencia sobre la eficiencia de cosecha de forraje por unidad de tiempo. Los antecedentes de estudio del efecto de la AF sobre el CMS no han incluido hasta el momento la variable tiempo de acceso a la pastura (Delagarde y O'Donovan, 2005). El conocimiento de estas relaciones permite establecer estrategias de alimentación donde se logran buenas eficiencias de cosecha y se minimizan los efectos negativos del animal sobre la pastura (Chilibroste et al., 2007).

\section{CONCLUSIONES}

La aplicación de medidas de manejo controlando el FD y $\mathrm{H}$ al momento de entrada y salida del pastoreo significaron en promedio 6,4 $\mathrm{kg} \mathrm{MS} \mathrm{ha}^{-1} \mathrm{~d}^{-1}$ extra producidos, lo que implicó una producción adicional total de $2336 \mathrm{~kg}$ $\mathrm{MS} \mathrm{ha}^{-1} \mathrm{año}^{-1}$. El mayor impacto de estas medidas fue en el otoño que explicó el 52\% del incremento total.

La AF, el tiempo de acceso a la pastura, FD y H, explicaron el $75 \%$ de la variación observada en $\mathrm{D}$ en cada sesión de pastoreo.

La alta asociación entre AF y D (>70\%) permite ubicar a esta variable como factor crítico en el manejo de la alimentación en general y de las pasturas en los sistemas pastoriles de producción de leche.

\section{REFERENCIAS}

Berretta, E., Guerra, J., De Mattos, D., 1993. Registros físicos en la producción pecuaria. Unidad de Difusión e Información Tecnológica del INIA, Montevideo, Uruguay, INIA, Serie Técnica 39, pp. 1- 28.

Chilibroste, P., Gibb, M.J., Soca, P., Mattiauda, D.A., 2015. Behavioural adaptation of grazing dairy cows to changes in feeding management: do they follow a predictable pattern?. Animal Production Science 55, 328-338.

Chilibroste, P., Battegazzore, G., 2014. Proyecto de Producción Competitiva. Informe técnico Cooperativa Nacional de Productores de Leche (CONAPROLE), Montevideo, Uruguay, pp. 1-31.

Chilibroste, P., Soca, P., Mattiauda, D.A., 2011. Balance entre oferta y demanda de nutrientes en sistemas pastoriles de producción de leche: potencial de intervención al inicio de la lactancia, en: XV Congreso Latinoamericano de Buiatría - XXXIX Jornadas Uruguayas de Buiatría. Paysandú, Uruguay, pp. 91-96.

Chilibroste, P., Soca, P., Bentancur, O., Mattiauda, D.A., 2010. Estudio de la conducta en pastoreo de vacas Holando de alta producción: síntesis de 10 años de investigación sobre relación planta animal suplemento en la Facultad de Agronomía-EEMAC. Agrociencia Uruguay 3, 101-106.

Chilibroste, P., Gibb, M., Tamminga, S., 2005. Pasture characte- ristics and animal performance, in: Dijkstra, J., Forbens, J., France, J. (Eds.), Quantitative aspects of ruminant digestion and metabolism, $2^{\text {nd }}$ edition. CAB International. Wallingford, UK. pp: 681-706.

Chilibroste, P., Ibarra, D., Zibil, S., Laborde, D., 2003. Proyecto Interacción Alimentación-Reproducción, Informe final 2003. Informe Técnico Área Producción Lechera y RR.CC., Cooperativa Nacional de Productores de Leche (CONAPROLE), Montevideo, Uruguay, pp. 1-52.

Delagarde, R., Peyraud, J.L., Parga, J., Ribeiro, H., 2001. Caractéristiques de la prairie avant et après un pâturage: quels indicateurs de l'ingestion chez la vache laitière?. Rencontres Recherches Ruminants 8, 209-212.

Delagarde, R., O’Donovan, M., 2005. Modelling of herbage intake and milk production by grazing dairy cows, in: Utilization of grazed grass in temperate animal systems. Proc Satellite Workshop $20^{\text {th }}$ International Grassland Cong. Dublin, Ireland, pp. 89-104.

Dirección de Estadísticas Agropecuarias (DIEA), 2014. Estadísticas del sector lácteo 2013. Serie de Trabajos Especiales $\mathrm{N}^{\circ} 324$, pp. 1-44. https://www.google.com.uy/ur

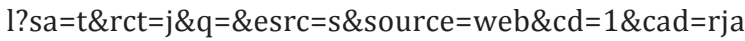
\&uact=8\&ved=0ahUKEwiqqbuBtrPOAhUEHJAKHbCfC d0QFggaMAA\&url=http\%3A\%2F\%2Fwww.mgap.gub. uy $\% 2$ Fportal\%2Fafiledownload.aspx\%3F2\%2C5\%2C1 04\%2C0\%2CS\%2C0\%2C9878\%253BS\%253B2\%253B 26\%2C\&usg=AFQjCNEydSwCDG6NO6eYhLUnbFA5ad7 fpg\&bvm=bv.129389765,d.Y2I

Dillon, P., Roche, J.R., Shalloo, L., Horan, B., 2005. Optimising Financial Return from Grazing in Temperate Pastures. Utilization of Grass in Temperate Animal Systems, in: Murphy, J.J. (Ed.) Proceedings of a Satellite Workshop of the XXth International Grassland Congress, Cork, Ireland. Wageningen Academic Publishers, the Netherlands, pp. 131-148. http://dx.doi.org/10.3920/978-908686-554-3.

Formoso, F., 2009. Aspectos a considerar para mejorar la producción y utilización de forraje durante otoño e invierno. Instituto Nacional de Investigaciones Agropecuarias (INIA) La Estanzuela, Revista INIA N 17. Colonia, Uruguay, pp. 41-47.

Formoso, F., 2008. Principales variables a considerar para aumentar la eficiencia de la producción y utilización de forraje, especialmente durante otoño e invierno. Instituto Nacional de Investigaciones Agropecuarias (INIA) La Estanzuela, Serie Actividades de Difusión 532. Colonia, Uruguay, pp. 1-20.

García, S., Fariña, S., 2010. ¿Hasta dónde intensificar en sistemas "extensivos" de producción de leche? Parte 1 y 2. Manejo de pasturas y suplementación. XXXVIII Jornadas Uruguayas de Buiatria, Paysandú, Uruguay, pp. 52-59.

Hydock, K.P., Shaw, N.H., 1975. The comparative yield method for estimating dry matter yield of pasture. Australian Journal of Experimental Agriculture and Animal Husbandry 15, 663-670.

Mayne, C.S., Wright, I.A., Fisher, G.E.J., 2000. Grassland Management under grazing and animal response, in: Hopkins, A. (Ed.), BBSRC Institute of Grassland and Environmental Research. Grass: Its production \& utilization, Devon, UK, pp. 247-286.

Ministerio de Ganadería Agricultura y Pesca - Recursos Natu- 
rales Renovables (MGAP-RENARE), 1976. Carta de Reconocimiento de Suelos del Uruguay - Escala 1:1.000.000. http://cebra.com.uy/renare/media/Carta-de-reconocimiento-de-Suelos-del-Uruguay-1.1.000.000-imprimirA0.pdf

Parga, J., Peyraud, J.L., Delagarde, R., 2000. Effect of sward structure and herbage allowance on herbage intake and digestion by strip-grazing dairy cows, in: Rook, A.J., Penning, P.D., (Eds.), grazing management: the principles and practice of grazing, for profit and environmental gain, within temperate grassland systems. BBSRC Institute of Grassland and Environmental Research, Devon, UK, pp. 61-66.

Parsons, A.J., Chapman, D.F., 2000. The principles of pasture Growth and Utilization, in: Hopkins, A., (Ed.), BBSRC Institute of Grassland and Environmental Research. Grass: Its production \& utilization, Devon, UK, pp. 31-79.

Parsons, A.J., 1988. The effect of season and management on the growth of grass swards, in: Jones, M.B., Lazenby, A., (Eds.), The grass crop. The physiological basis of production. Springer. Netherlands. pp. 129-177.

Pérez-Prieto, L.A., Delagarde, R., 2012. Meta-analysis of the effect of pregrazing pasture mas son pasture intake, milk production and grazing behaviour of dairy cows strip-grazing temperate grassland. Journal of Dairy Science 95, 5317-5330.
Peyraud, J.L., Comeron, E.A., Wade, M.H., Lemaire, G., 1996. The effect of daily herbage allowance, herbage mass and animal factors upon herbage intake by grasing dairy cows. Annales de Zootechnie 45, 201-217.

Prache, S., Peyraud, J.L., 1997. Préhensibilité de l'herbe chez les bovins et les ovins. Inra Productions animales 10, 377-390.

Rook, A.J., 2000. Principles of foraging and grazing behavior, in: Hopkins, A., (Ed.), BBSRC Institute of Grassland and Environmental Research. Grass: Its production \& utilization. Proceedings of the British Grassland Society, Devon, UK. pp. 229-246.

Rossi, J.L., García, S.C., 2006. ¿Cuál es el piso de la producción pastoril?. Desarrollo argentino, 129, 51-53. http:// www.agro.uba.ar/sites/default/files/catedras/piso.pdf SAS. 2010. Institute Inc. SAS/STAT. User's guide, Carey, North Caroline, USA.

Wales, W.J., Stockdale, C.R., Doyle, P.T., 2005. Plant and sward characteristics to achieve high intake in ruminants, in: Murphy, J.J., (Ed.), Utilisation of grazed grass in temperate animal systems, proceedings of a satellite workshop of the XX ${ }^{\text {th }}$ International Grassland Congress. Wageningen Academic Publishers, Cork, Ireland, pp. 37-48.

Zanoniani, R., 2010. Estado actual del conocimiento en producción de pasturas, brecha tecnológica. Agrociencia Uruguay 14(3), 26-30. 
